\title{
BMJ Open Older adults' understandings and perspectives on frailty in community and residential aged care: an interpretive description
}

\author{
Mandy Archibald (D) , ${ }^{1,2,3}$ Michael Lawless (D) , , Rachel C Ambagtsheer (D) ,3,4 \\ Alison Kitson ${ }^{1,3}$
}

To cite: Archibald M, Lawless M, Ambagtsheer RC, et al. Older adults' understandings and perspectives on frailty in community and residential aged care: an interpretive description. BMJ Open 2020;10:e035339. doi:10.1136/ bmjopen-2019-035339

- Prepublication history for this paper is available online. To view these files, please visit the journal online (http://dx.doi. org/10.1136/bmjopen-2019035339).

Received 31 October 2019 Revised 15 January 2020 Accepted 29 January 2020

Check for updates

(c) Author(s) (or their employer(s)) 2020. Re-use permitted under CC BY-NC. No commercial re-use. See rights and permissions. Published by BMJ.

${ }^{1}$ College of Nursing and Health Sciences, Flinders University, Bedford Park, South Australia, Australia

${ }^{2}$ College of Nursing, University of Manitoba, Winnipeg, Manitoba,

Canada

${ }^{3}$ Centre of Research Excellence in Frailty and Healthy Ageing, Adelaide, South Australia, Australia

${ }^{4}$ Health, Torrens University Australia, Adelaide, South Australia, Australia

Correspondence to Dr Mandy Archibald; mandy.archibald@umanitoba.ca

\section{ABSTRACT}

Objectives Despite growing interest in frailty as a significant public health challenge, comparatively little is known about how older adults perceive and experience frailty, limiting the effectiveness of strategies to improve frailty management and prevention. The objective of this study was to understand how older people, including frail older persons in residential aged care, perceive and understand frailty through an interpretive-descriptive qualitative study.

Setting Aged care facility, community-based university for older persons and an aged care auxiliary care group in a large metropolitan centre in South Australia.

Participants 39 non-frail, prefrail, frail and very frail South Australian older adults.

Methods Seven focus groups were conducted. Participants completed one of two frailty instruments depending on setting and indicated whether they selfidentified as frail. Data were analysed inductively and thematically by two independent investigators.

Results Frailty was described according to three schemas of (1) the old and frail: a static state near the end of life; (2) frailty at any age: a disability model; and (3) frailty as a loss of independence: control, actions and identity. In addition, a theme was identifying linking mindset, cognition and emotion to frailty. The term frailty was viewed negatively and was often implicated with personal choice. There was little correlation between frailty assessments and whether participants self-identified as frail.

Conclusions Aside from a disability model, views of frailty as unmodifiable permeated older persons' diverse perspectives on frailty and are likely to impact health behaviours. To our knowledge, this is among the largest qualitative studies examining consumer perceptions of frailty and contributes a clinically relevant schema linking age, prevention and modifiability from a consumer perspective.

\section{INTRODUCTION}

Medical literature is rife with references to the burdens and challenges presented by an ageing population, such as the higher incidence of chronic disease in aged populations, sensory and physiological changes
Strengths and limitations of this study

- Inclusion of non-frail, prefrail, frail and very frail older adult participants from community, assisted living and residential aged care settings contributes a range of comparative perspectives on frailty sensitive to context, age, and frailty status.

- This study involves an in-depth interpretive analysis to contribute a clinically relevant schema linking perceptions of frailty with perceptions of the prevention and modifiability of frailty from a consumer perspective.

- A unique contribution of this research is the inclusion of objective measures and self-assessment of frailty status, which provides insight into self-perception with potential implications for health behaviours including frailty screening.

- Despite a large qualitative sample, our sample under-represented non-English speaking people from culturally and linguistically diverse backgrounds and those living in rural regions who may hold different perceptions.

impacting self-management and corresponding socioeconomic implications of societal ageing. ${ }^{1}$ Increasingly common among these references is frailty, a state of increased risk for adverse health outcomes such as falls, dependency, institutionalisation and mortality. ${ }^{2}$ Responding to the clinical challenges presented by frailty, particularly in light of international prevalence ranges of $4.9 \%-27.4 \%$ for frailty and from $34.6 \%$ and $50.9 \%$ for prefrailty, ${ }^{3}$ requires coordinated and multipronged approaches to management. ${ }^{45}$ Although attending to consumer perspectives would optimise the effectiveness of frailty education, screening and management strategies, how older adults perceive frailty is under-represented in the frailty literature. While qualitative studies of differing focus have been conducted in the $\mathrm{UK}^{6-9}$ the Netherlands, ${ }^{10}$ Europe $^{11}$ and the USA, ${ }^{12}$ no 
empirical data exist on the perspectives of older adults in Australia. ${ }^{13}$ This study contributes knowledge to this area by examining how older persons along the frailty trajectory perceive and experience frailty in order to inform knowledge translation and service improvement initiatives in a Centre for Research Excellence (CRE) in Frailty and Healthy Ageing in Australia.

Existing research has characterised the experiences of living with frailty as highly nuanced, involving complicated and enduring transitions in health and illness. ${ }^{14-16}$ Older people's understandings of frailty are often multidimensional, encompassing complex and interacting physical, social and psychological factors. ${ }^{17-19}$ Older adults living with frailty may differentiate between the emotional (ie, feeling frail) and physical (ie, being frail) experiences of frailty, wherein being frail is seen as an imposed medical classification and feeling frail may reflect the psychosocial impacts of age-related disability, physical impairment or traumatic life events. ${ }^{20}$ Older people often resist frailty as an identity, even when meeting objective frailty criteria. ${ }^{6}$ Actively resisting a frailty label may help preserve a positive identity in the face of age-related functional decline and illness experience and prevent negative outcomes such as social isolation, reduced physical activity and depression. ${ }^{621-24}$ These findings suggest a strong interdependency between frailty perspectives, the construction of identities in older age, and strategies to maintain a selfperception of wellness, strategies that in turn can be identified and supported. ${ }^{6}$

Older adults' understandings of frailty are often inconsistent with evolving medical views of the condition. ${ }^{12}$ As a result, health behaviours and patient healthcare system interactions may be affected in unanticipated ways. ${ }^{62}$ While more comprehensive representations of stakeholder experiences and understandings have been recommended, there is limited research on this topic, and frail older persons and those in residential care are often excluded from the research, reflecting critical information gaps. In this study, we inquired into older persons' perceptions and understandings of frailty in what is, to our knowledge, the largest qualitative study on this topic. We build on current knowledge by exploring perspectives across frailty subgroups (ie, non-frail, prefrail, frail and very frail: categorical determinants stipulated through selection of assessment tools) from older persons in various contexts and care environments to illuminate implicit assumptions, beliefs, fears and constructs around frailty. We explore how these views impact perspectives towards frailty prevention and management, in order to inform knowledge translation initiatives and guide health service improvements. This research, along with concurrent research exploring perspectives on frailty and frailty screening among key healthcare provider groups, ${ }^{13}$ 26-28 will contribute to healthy ageing research and, ideally, policy in Australia, catalysed through the established channels of the CRE (eg, including advisory group members with state health and federal cabinet positions), with likely relevance to other jurisdictions.

\section{METHODS}

\section{Theoretical framing}

This research is outlined in the protocol developed by Archibald and colleagues, ${ }^{13}$ which describes a collaborative knowledge translation approach ${ }^{29}$ to understanding how older adults and healthcare providers perceive frailty and frailty screening, in order to refine the research problem and guide future research and translation efforts. This study is underpinned by a constructivist epistemological position. We were interested in how participants created and negotiated meaning around frailty while acknowledging multiple possible interpretations of participants' lived experiences. Our views were in part shaped through our experiences as qualitative and mixed methods researchers interested in how experience and perception influence health behaviours, a critical yet often overlooked component of applied health research.

\section{Design}

We conducted an interpretive descriptive qualitative study, which is an inductive approach appropriate for exploratory research and which emphasises the clinical application of qualitative data. ${ }^{30}$ Interpretive description encourages the use of metaphors as communicative devices arising from interpretive analysis. ${ }^{31}$ With assistance from site staff coordinators and administrators, participants were purposively sampled from community-based and residential care settings, including two different aged care providers and a continued learning university for adults aged over 50 years in metropolitan South Australia (population 1.3 million). These locations were chosen because they allowed a broad representation of non-frail, prefrail, frail and very frail older adults from diverse backgrounds, intended to represent the range of perceptions of frailty likely to be present among the wider study population, and because of alignment of the research objectives with the research priorities of the residential aged care facility.

\section{Ethics}

Participants provided free and informed written consent prior to the collection of demographic and qualitative data.

\section{Patient and public involvement}

Participants in our study were not directly involved in the setting of research questions or the data analysis. However, the study aim was derived from under-representation of consumer perspectives on frailty in the Australian context, and findings were directly used to inform dissemination and exchange strategies based on consumer preferences, understandings and identified needs. To aid dissemination, consumers were actively involved in the co-design of video resources used to communicate experiences and understandings of frailty (eg, https://www.youtube. com/watch?v=agGYvoLL_vo; https://www.youtube. $\mathrm{com} /$ watch?v=KzkIk94ysAg), as well as the participation 
in community-based events to catalyse frailty awareness based on study findings.

\section{Data collection}

Two members of the research team (MA and RA) collected data between February and April 2017. Demographic data were attained on birth year, gender, type of accommodation, suburb, and status as carer for another older person. Participants' perceived and objective frailty status was confirmed during demographic data collection by having participants indicate whether they identified as frail (ie, do you associate with being frail yourself?) and by use of one of two assessment tools. The FRAIL questionnaire screening assessment tool (a simple five-item assessment ${ }^{32}$ of Fatigue, Resistance, Aerobic capacity, Illnesses, and Loss of weight, wherein a score of 0 indicates robust health, a score of 1-2 indicates pre-frailty, and a score of 3-5 indicates frailty) was used for community-based participants. For participants in residential aged care, a care worker assessed degree of frailty status using the FRAIL-NH scale (Fatigue, Resistance, Ambulation, Incontinence, Loss of weight, Nutritional approach, and Help with dressing) ${ }^{33}$ wherein a score of $0-2$ is assigned across the aforementioned seven domains. A score of 2-5 indicates frailty, and score of 6-14 indicates most frail-a term that is used interchangeably here with very frail. Details of the focus group questions and data collection strategies are reported elsewhere ${ }^{13}$ but included seven semistructured focus groups with an interview length ranging from 69.51 to $93.32 \mathrm{~min}$ (mean $(M)=81.69 \mathrm{~min}$ ). Focus group questions generally proceeded from the general to the particular and included questions on the meaning of frailty, experiences of becoming frail, the relationship between frailty and ageing, the trajectory of frailty and questions related to the prevention and treatment of frailty, including frailty screening. Given the breadth and robustness of the data, currently presented findings focus on understandings and perceptions of frailty.

\section{Participants}

Criterion-based sampling was used to identify prospective participants from the three purposively selected sites. Recruitment was facilitated through administrators at the community-based sites who identified interested participants and through the recreation coordinator in the aged care facility. Physical and cognitive comorbidities of participants were not assessed.

\section{Data analysis}

Focus groups were audio recorded, professionally transcribed, cleaned and deidentified. NVivo software (version 11.2.2) and Microsoft Excel were used for data management and to facilitate analysis. MA and ML independently analysed data using thematic analysis as described by Braun and Clarke. ${ }^{34}$

We repeatedly read transcripts to gain a sense of the whole, generated initial codes and notes on preliminary impressions and used these understandings to develop an initial coding framework to help us identify recurrent patterns within and between focus groups. We tentatively defined and labelled themes as we continued to integrate theoretically derived codes. We rigorously questioned thematic categories and negative cases and used thematic maps to explore, interrogate and elaborate on relationships between or within themes and refine definitions of candidate themes. When necessary, we restructured relationships between codes and themes and reviewed preliminary findings in team meetings. Analysis was largely inductive but also moved from the general to specific using a synthetic approach to cross-verify our interpretations. ${ }^{35}$ Generally, each focus group was treated as the unit of analysis; attention was paid to the origins of each code, be it at the focus group or individual level, and relevant interaction data were noted (eg, agreement or dissention). Three authors contributed to reviewing and refining themes by independently checking themes and illustrative examples against the original data. Analytic rigour and trustworthiness were promoted through regular consultation with coauthors, in which interpretations of the data (identified codes, preliminary themes and representative extracts) were cross-examined and compared; questions were asked about negative cases and across subgroups to generate explanatory understandings; field notes and analytic memos were discussed, and extracts that best illustrated each of the themes were selected.

\section{RESULTS}

Thirty-nine participants partook in the seven focus groups (community-based $(\mathrm{n}=22)$ and aged care $(\mathrm{n}=17)$ ) participants, aged between 62 and 99 years $(M=80.6$ years; $S D=9.6$ years). The sample consisted of $7(18 \%)$ nonfrail, $13(33 \%)$ prefrail and $19(49 \%)$ frail participants (table 1). Of the 19 frail participants, 7 (37\%) qualified as most frail using the FRAIL-NH scale. Of the 22 participants living in community, $4(18.2 \%)$ self-identified as frail, with 13 (59\%) meeting criteria for prefrailty and 2 (9\%) screening positive for frailty. Of the 17 participants living in residential aged care, $8(47 \%)$ self-identified as frail, with $17(77.3 \%)$ screening positive for frailty $(\mathrm{n}=10$; $58.8 \%)$ and most frail $(n=7 ; 41 \%)$.

Across the focus groups, many participants were familiar with the term frailty, drawing from personal experiences with and exposures to frailty as the basis for their knowledge. Frailty had not been thought about extensively and often lacked a specific meaning. Understandings of frailty varied significantly, with participants describing frailty as intertwined with old age (eg, 'the frail aged'), mental wellness and attitude, disability and dependence. Participants across community and residential care settings differentiated between these different 'types' of frailty. Although we did not observe systematic differences in whether participants emphasised the mental or physical aspects of frailty, we did observe three main ways that frailty was described by our sample: (1) the old and frail: a static state near the 


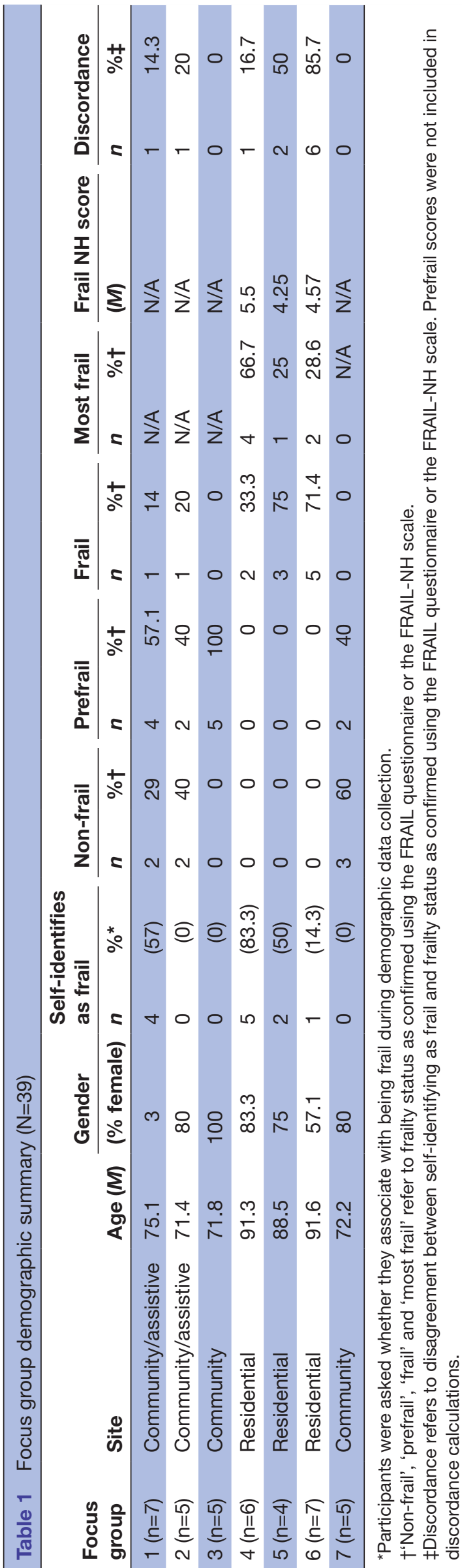

end of life; (2) frailty at any age: a disability model; and (3) frailty as a loss of independence: control, actions and identity. Mindset and cognitive/emotional frailty were discussed as important influencing factors cutting across the schema, were understood as either protective or as a type of frailty, were entwined with the concepts of attitude and choice and are indicated as a mediator under a distinct thematic heading. We viewed the schemas as abstracted conceptual structures containing general knowledge about frailty perceptions. ${ }^{36}$ References to specific focus groups are made using the abbreviation FG throughout the presentation of the results.

\section{The old and frail: a static state near the end of life}

Participants who related to frailty as a domain of the old and visually frail generally described frail persons as slow moving, skinny, hunched over, dependent on mobility aids and displaying characteristic behaviours and attributes. As one community-based participant expressed 'it's a physical picture ... how they walk and how they stand and even how they have a conversation sometimes' (FG2, male), to which the group extended with other examples such as unsteady gait or stance, and losing 'strength of speech'. Visually recognising frailty was central to representations within the 'old and frail' schema.

Despite the age and frailty status of participants in our sample, it was uncommon for participants to view themselves as old and frail. 'Old' typically referred to advanced age of 85 years and older, and age was viewed relatively; participants frequently compared themselves with someone older and less robust. As one participant stated, 'I've always associated frailty with age. I don't mean our age, I mean 85 plus' (FG3, female). This relativism impacted whether someone self-identified as frail; $69 \%$ of participants $(n=27)$ did not self-identify as frail, but of these, $37 \%(\mathrm{n}=10)$ met criteria for frailty using the frailty screening instrument administered at the beginning of the focus group. As one female participant in the community expressed: 'Frail ... I don't feel frail. I always think of frail people as much thinner than me, much older than I am. All those frail people of my youth were... they're probably around about where I am now actually, the age that I am now but they seemed very old when they were in their 70's' (FG7). One additional participant in robust health associated with being frail, a phenomenon that we termed 'negative discordance'.

The schema of the 'old and frail' was connoted with metaphors of end of life, fragility and being broken. This sentiment was expressed by a male carer in the community: '[frailty is] ... getting close to a trip to the cemetery ... I think it [frailty] has that connotation ... it almost feels as if you're dropping a cup on the ground to smash... it's almost broken' (FG2, male). Within this perspective, frailty was entered near the end of life and, once reached, was unlikely to be improved on. This contributed to negative perceptions of frailty as a label because of the perceived unmodifiability of frailty. Frailty was characterised by fragility and, 
aligned with the metaphor of a cup smashing on the floor, was difficult if not impossible to repair.

Physical representations of frailty were widespread, illustrated by quotes such as 'to me frailty has always been body' (FG2, female), stemming from participants visually recognising frail persons in their lives. However, some participants struggled with the word frailty because it suggested an emphasis on the physical body rather than mental components, which were also regarded as important. As one female participant in the community expressed: 'I have problems with the word frailty because we always tend to associate it with physical things and not mental things' (FG3). Although there was assentation within the focus group following this statement, participants disagreed on the precise role of mental components. Communitydwelling participants were more likely to acknowledge a mental component to frailty but often associated mental aspects with a lack of motivation, strength or resolve. This was captured in a discussion between community-dwelling participants (FG3) as follows:

Female 1: Is there such a thing as a frail mind? I was thinking of...

Female 2: Very good.

Female 1: Someone lacking motivation maybe, would you call that a frail person?

Female 3: ... I thought that's an excellent...

Female 2: I think that's possibly true because if you have no main motivation then you eventually, you give up doing things.

The concept of giving up on doing things because of a perceived link between motivation and mental frailty is further explored under the heading 'mental state influences perspectives on frailty'.

\section{Frailty at any age: a disability model}

Many participants, particularly non-frail and prefrail persons in community settings, regarded frailty as synonymous with disability. Frail and very frail participants from aged care were less likely to hold this perspective. Within this schema, participants were more likely to view frailty as possible at any age and often related the onset of frailty to a critical stressor event. Frail persons were recognisable by specific impediments or disabilities, which often occurred following a critical stressor event (eg, car accident) and at any age. As a female participant in the community expressed: 'There was a child who broke their spine diving into a swimming pool as the age of 13. Now she's frail because she's permanently in a wheelchair and I have another friend who's grandmother is 99, she's anything but frail' (FG1). Other participants echoed this response and emphasised that frailty is not necessarily tied to age because people can be frail all their lives (eg, FG3 and FG7).

The disability perspective was aligned with a metaphor of a mosaic rather than a cup smashing wherein one aspect of a person could be frail while other aspects of a person remained unaffected. As one participant expressed, 'some are frail in some areas and not in others... every individual can be frail or strong in different ways' (FG6). As such, within this schema, there was less of a tendency to link frailty with identity or the end of life. Mental aspects were included as possible domains in which a person might experience and exhibit frailty; participants often viewed frailty as involving distinct or interrelated mental and physical subtypes with either one or both occurring in an individual. Here, frailty was thought of as a combination of mental and physical frailty (FG1, FG2, FG5 and FG7), as physical only (FG1), or as more mental than physical (FG3). A cyclical relationship between mental and physical frailty was often identified (FG3).

Perspectives of frailty as a dynamic state were common within the disability view

Frailty was seen as a dynamic state (FG1, FG7) or continuum more often when associated with a disability or acute life event, and possible at any age. In contrast to the old and frailty model, within this schema, transitioning in and out of a frailty state was generally accepted as 'you can go through periods of life where you are frail' (FG7). As one participant shared, 'I had to go into hospital because I had pancreatitis and so I was frail because I was in hospital for six months and I couldn't walk afterwards but I have got the use of my legs and everything back now. So I was frail but now I'm less frail' (FG1). Participants acknowledged the role of specific lifestyle choices in preventing frailty. Of these, exercise was the most commonly mentioned. Nutrition was also frequently acknowledged.

Frailty as a loss of independence: control, actions and identity When discussed as a loss of independence, frailty related to what a person can do and also who a person is and has been all of their lives. This perspective was also associated with frailty as a static state associated with old age. Perceptions of control over oneself and one's environment were paramount to this schema.

Whether a person was able to do things for themselves was a defining attribute of this frailty schema. As such, a persons' ability was the benchmark for frailty. As one participant simply stated: 'I don't think of frail... if you're still capable of doing things' (FG3, female). However, ability was often considered in relation to a persons' past capability (eg, 'you can't do what you used to do' (FG7, female); 'A frail person is not able to conduct themselves as they have all their lives' (FG5, male), and also future capacity. In this way, a person's ability was closely linked to trajectory (ie, what a person was able to do in the past and what the future holds) and included notions of control over ones own body and surrounding environment.

Frailty is seen as a loss of control over oneself and one's environment and is closely tied to mobility

Participants across all focus groups linked frailty with mobility and drew strong associations between mobility and independence. The mobility-independence link was 
expressed as follows, 'Frailty as a little person in stature trying her darndest to push the walker or whatever and lose all independence that she had... or she or he had' (FG2, male). An emphasis on visually recognising frailty was pervasive.

The mobility and independence interdependency was often implied as antecedents to 'control', specifically control over one's body and the environment. The inability to move freely was central to this relationship. As one community dwelling participant expressed, 'That would be my concept of reaching that stage of frailty where you don't have the freedom of movement' (FG7, male), and it was commonly expressed that if frail, 'you'd no longer be in control of yourself' (FG2, female).

Other times, the relationship between loss of control and frailty included mental dimensions alongside a strong physical component. As a community-dwelling participant explained: 'Frailty is being unable to control your environment. What I'm saying there is that in youth you believe you're able and most times you are able to control situations as they occur around you ... then sort of frailty comes into it when sort of your own body is saying that I can no longer do these things or I can't remember ...' (FG7, male). In the context of independence, losing control also meant having no control over what is lost or taken away. As a male carer expressed, 'independence is going to be taken away cause you're not able to do things' (FG2). Loss was something that happened to a person, an undesirable event.

Mobility was central to the discussion of independence. Mobility aids helped participants visually recognise a frail person. Discussions of mobility centred on mobility aids such as frames, walkers or wheelchairs and less commonly speed. Other factors impacting mobility such as rehabilitation or pain associated with disability were infrequently mentioned. In this way, mobility devices were frequently equated with frailty as an object signifier. As one community-based participant expressed, 'I see that as frailty, you know once you've got a walking stick or a frame. That's it' (FG3, female). Mobility was also discussed as a trajectory beginning with using a mobility aid and eventually progressing through to other aids. The type of mobility aid was again a signifier of frailty status: 'Well just say once a person is in those princess chairs they must... you know, my sister's in one of those so I guess she's frail. I mean she can't walk or anything now', to which another participant conferred 'It's a sign of frailty' (FG6, female).

\section{Frailty and a loss of independence is linked to identity and self-}

\section{worth}

It was common for participants who discussed frailty as a loss of independence to link frailty with identity and self-worth. At times this was expressed overtly in relation to individuality. As one community-based participant expressed: 'That whole kind of independence and I'm an individual kind of is lost and I think that relates to frailty' (FG2, male). Others in the focus group shared this sentiment, emphasising the strong link between independence and identity: 'for now someone to come in and say, well I now have to shower or I now have to do your medication, you've lost a bit of your independence that's made you, you' (FG2, female). Independence and individual actions contribute to who a person is and what a person can express about oneself; losing these capabilities therefore jeopardised ones sense-of-self: 'They seem to lose that kind of strength of their speech and they become kind of almost withered and you feel somehow or other that they've lost their self ...' (FG2, male).

The link between independence, identity and self-worth persisted with frail and very frail participants in residential care. The loss of independence signified the end for a male participant who expressed: '[frailty] means the end of everything because you can't do anything' (FG4, male). Other times, the term frailty was perceived as an infraction on self-worth and was suggestive of shame. As a female participant from residential care expressed, '[frailty] means you're not good enough to have anything' (FG4, female). Within the residential care environment in particular, discussing frailty conjured negative feelings associated with age-related change, with some participants becoming emotional. This led the interviewer to refocus the focus group discussion on positive aspects of ageing and undertake activities aligned with the Health Research Ethics Board-approved protocol for adverse events. This included validating the issue, allowing the participant time to recover or the option of leaving the interview, and contacting the participant with an additional support person if deemed necessary. In this circumstance, validation and support through active listening during the focus groups were the only protocol steps required.

\section{Mediating factor: frailty is influenced by mental state and attitude}

Participants in every focus group spoke about some aspect of mental frailty, attitude or mental state as important to physical frailty. Participants generally regarded attitude and mental state as either protective factors or as causes of frailty. Through analysis we identified two dominant patterns pertaining to the relationship between mental state and attitude with frailty.

Within the 'old and frail' schema, mental state and attitude are seen as protective towards frailty but are entangled with choice and individualism

Participants that associated frailty with advanced age generally acknowledged that not everyone becomes frail. There was a pervasive belief outside of the disability schema that mindset, mental strength and attitude distinguished those people who became frail from those who did not. Mindset and attitude were understood as how a person perceives himself or herself and adapts to life and age-associated change. The role of attitude and resilience underlay discussions about if and how frailty occurs and progresses and was particularly common for participants in community-based settings. By having strength of mind, not giving in or putting in the effort, frailty could 
be slowed or prevented (eg, FG2 and FG3). Not all participants agreed with the assessment that people choose to be frail but often focused on the importance of coping and individual resilience to frailty.

It was generally agreed on that ageing brings changes that are at times difficult to cope with. To cope with these changes and avoid frailty, participants expressed the 'need to be strong willed to not fall at first hurdle' (FG2, male). In residential care environments, people were confronted with their changing abilities, and it was regarded as important to keep positive and find enjoyment with an eye on the future: 'you can't look back. Got to look to the future' (FG4, female). Acceptance of agerelated change was integral to finding a way forward.

Accepting and moving forward were entangled with the notion of choice, thereby creating an individualistic picture of how frailty and ageing occur. The alternative to coping effectively and identifying new ways forward was to choose frailty. At times, the role of choice in frailty was overtly expressed, in discussing whether frail persons choose to be like that (FG3). Some participants expressed that people can 'let themselves get frail' (FG2, female) and enjoy being frail for the attention. As one participant stated: 'some people do like being frail because they have people coming ... then they have people giving them attention, perhaps they're lacking' (FG2, female). There was a sense that people give up, by just 'sitting back and doing nothing' (FG3, female). As a community-based participant expressed, 'I think they're not frail as we're talking about. I think they've just given up' (male), to which another participant agreed. Choice was also expressed as a mindset (eg, FG3, female).

When choice was more implicit, participants emphasised a decision point around whether age-related change could be accepted in order to move forward with strength of mind. A male participant in the community who had cared for his recently deceased partner captured this sentiment: 'it would really take a strength of mind to say hey, this is the world I'm in now. I can't do what I used to do. Let's be grateful for what I've got and see if I can explore other avenues ... that's what this is all about isn't it? It's really all about if you were put in that situation, how would you cope? How would you respond? And how's the way forward?' (FG2). Keeping busy was intimately tied to acceptance, and various activities were mentioned.

Participants also discussed which choices could lead to different outcomes (and how) over the course of ones' life. For instance, modifiable factors, such as making good dietary selections, exercising regularly and dealing with illness well were seen as positive life choices linked to attitude, that create protective habits throughout life. Exercising the mind was also important, as a female participant from residential care expressed: 'We have to exercise our minds as well as our bodies because if we don't we get into a state of... what would you call it... non compos mentis' (FG6).
Mental frailty: attitude and mental state as a cause or type of frailty Participants who acknowledged mental frailty regarded it as distinct from or coexisting with physical frailty (ie, precipitated by or precipitating). This was observed across community-based and residential care settings, wherein participants discussed coexisting subtypes of frailty and often were in disagreement about whether frailty denoted a purely physical condition or whether it included a mental aspect. As one male participant explained: 'There are two types of frailty, mental and physical... most people don't become mentally and physically frail at the same rate... the differential between physical frailty and mental frailty. When you've got both you're in trouble' (FG5, male).

Numerous questions were raised about the nature of mental frailty. Some participants implicated mental frailty with depression, at times treating the two concepts as synonymous. As one participant stated, 'It's more of a mental issue isn't it, I think. Depression, that's what my first thought is...' (FG3, female). Frailty was then discussed following this participant generated question: 'is frailty depression and is depression frailty in your mind?' to which the group responded with interest. Generally, discussions of mental frailty circled back to concepts related to strength of mind, attitude and choice, and so in comparison, physical frailty was regarded as 'more real' than mental frailty.

Other participants expressed that mental frailty in essence 'caused' physical frailty. Community-based participants stated, 'they become physically frail because they've been mentally frail' (FG2, female), or 'if you've got a disparity in your mental ability, then you create this frailty and then you've got the physical side as well' (FG2, male). However, mental frailty was more associated with 'giving up' and had worse connotations than physical frailty. Compared with physical frailty, mental frailty was regarded as 'more horrifying to the person' (FG2, male) or something that people are sensitive about. As one female participant stated: 'I think frailty is fine if it's just talking about physical frailty but when it's mental frailty people don't want to admit they're frail. So perhaps another word needs to be thought of as far as the mental state goes but I don't know what because people are very sensitive about that' (FG7).

\section{DISCUSSION}

We identified three dominant schemas for how older persons view frailty including a model of frailty as old age, wherein frailty was largely unpreventable and unmodifiable; a disability model, wherein frailty was modifiable and could occur at any age; and an independencefocused model, associated with loss of ability, control and identity. Mental wellness implicated each schema. Choice and individualism permeated discussions of mental frailty more often than physical frailty and suggested a broader stigmatisation of mental health issues. 
Extending UK, ${ }^{6-9}$ the Netherlands, ${ }^{10}$ European $^{11}$ and American ${ }^{12}$ literature on older persons' perceptions and experiences of frailty this is the first study examining older persons' perceptions of frailty in Australia and the largest consumer study to date on this topic. In contrast to other studies, we included perspectives of very frail older persons residing in residential aged care in order to strengthen the breadth of participant perspectives, which are likely to differ based on lived experience of frailty. We compared participants' self and objective frailty assessments to generate insight into the complex interplay between self-perception, frailty connotations and experience, thereby aligning with international research programmes using common operational definitions of frailty. Although we included people with a range of frailty statuses, our sample under-represented nonEnglish speaking people from culturally and linguistically diverse backgrounds and those living in rural regions who may hold different perceptions. We did not assess comorbidities that could also have influenced participant perceptions.

Although frailty was not unfamiliar to participants, its meaning was vague and contingent on direct (eg, seeing visibly frail people) and indirect (eg, media portrayals) exposures to frail and very frail persons in their personal lives. As such, participants frequently associated frailty with end of life, contributing to the differences between consumer and medical understandings of frailty. Such differences have been discussed elsewhere ${ }^{6} 712$ along with the physical, psychological ${ }^{12}$ and social dimensions of frailty ${ }^{9} 1719$ replicated in our study. Participants also routinely resisted self-identifying as frail, a practice previously associated with positive outcomes. ${ }^{6} 72037$ Although research demonstrates the positive impact of mindset on health outcomes, ${ }^{38-40}$ the emphasis on individual choice in our study, particularly as it related to choice and 'mental frailty', may reflect buried ageist perspectives and internalised social biases around mental health, positioning mental ailments as 'less real' than physical ailments. ${ }^{18}$

Previous research shows that frail older adults may be more open to discussing frailty than their non-frail and prefrail peers, ${ }^{12}$ although irrespective of frailty status, 'frailty' is not a term with which most older adults identify. ${ }^{12}{ }^{41}$ Our research shows that negative perspectives of frailty are reinforced when frailty is viewed as unavoidable, unmodifiable and associated with end of life, which diminishes the perceived relevance of frailty to less frail participants. This finding is relevant to quantitative research demonstrating an interaction between negative perceptions of ageing and frailty in predicting future frailty, cognitive function and other health changes when controlling for factors including age, gender, socioeconomic status, depressive symptoms and baseline frailty. ${ }^{242}$ Furthermore, there is an established body of work linking self-perception of ageing more generally with a range of health outcomes for older people. ${ }^{38-40}$ Negative perspectives towards ageing and frailty can limit engagement with health system initiatives ${ }^{1724}$ and suggest that frailty identification and management strategies could be optimised through positively framed education and public awareness tailored to consumer perspectives, misconceptions, and strategies that focus on independence and control-the precipices of motivation for consumers in our study. The three schematic representations generated through this inquiry provide nuanced considerations for how frailty screening, education, and treatment could be tailored to better align with consumer perspectives, creating opportunity to maximise satisfaction with and benefit of frailty prevention and management strategies. Such considerations are further explored in an affiliated manuscript focused specifically on frailty screening. ${ }^{26}$

This research was conducted within a Frailty and Healthy Ageing CRE and is being used to inform future research and codesign educational interventions, including videobased resources (https://www.youtube.com/watch?v= agGYvoLL_vo; https:/ / www.youtube.com/watch?v=KzkI$\mathrm{k} 94 \mathrm{ys} A g)$. The perspectives identified through this and affiliated research projects have shaped corresponding presentations to state and federal political representatives (eg, Minister of Health) and have informed associated health service delivery proposals of the CRE in efforts to shape health policy. In addition to their clinical utility, the three schemas identified can inform future qualitative (eg, in-depth exploration of clinical communication and decision making) and quantitative research (eg, testing conceptual models of frailty and correlations to clinical outcomes) and inform targeted clinical service provision. Further research is needed to understand the relationships between a frailty identity and particular health behaviours and outcomes, whether targeting misconceptions and stigma around frailty can mitigate fear and information avoidance and how healthcare provider communication can impact older persons readiness to participate in frailty and healthy ageing strategies. Public awareness strategies to reduce frailty and mental healthrelated stigma continue to be needed.

\section{Dissemination declaration}

We have used findings from this research to inform a series of resources used to actively share, exchange and disseminate findings to research participants and the public. These have included community-based presentations, research-based performance and art exhibitions, and numerous videos representing consumer experiences and perspectives on frailty and healthy ageing.

Twitter Mandy Archibald @Mandy_Archibald and Michael Lawless @mt_lawless

Acknowledgements MA acknowledges the fellowship support received from the Canadian Institutes of Health Research. We would like to thank the various participants who shared so openly with us.

Contributors MA conceptualised and led all components of the study. ML and RCA contributed to data analysis and manuscript writing. AK contributed to study conceptualisation, data analysis and writing. All listed authors meet criteria for authorship.

Funding This research is supported by the National Health Medical Research Council Centre of Research Excellence grant 'Frailty Trans-disciplinary Research To Achieve Healthy Ageing' (GNT 1102208) and a GTRAC-Resthaven Research grant. 
Funders provided financial support for this research but were not involved in how the research was conducted.

Competing interests None declared.

Patient consent for publication Not required.

Ethics approval Ethics approval was granted from the University of Adelaide Human Research Ethics Committee (H-2016-238).

Provenance and peer review Not commissioned; externally peer reviewed.

Data availability statement Research participants did not grant permission for the data to be made publicly available.

Open access This is an open access article distributed in accordance with the Creative Commons Attribution Non Commercial (CC BY-NC 4.0) license, which permits others to distribute, remix, adapt, build upon this work non-commercially, and license their derivative works on different terms, provided the original work is properly cited, appropriate credit is given, any changes made indicated, and the use is non-commercial. See: http://creativecommons.org/licenses/by-nc/4.0/.

\section{ORCID iDs}

Mandy Archibald http://orcid.org/0000-0003-4767-1031

Michael Lawless http://orcid.org/0000-0002-2536-6442

Rachel C Ambagtsheer http://orcid.org/0000-0002-9391-6709

\section{REFERENCES}

1 National Institute on Aging. Why population aging matters. A global perspective. Available: https://www.nia.nih.gov/sites/default/files/ 2017-06/WPAM.pdf

2 Clegg A, Young J, lliffe S, et al. Frailty in elderly people. The Lancet 2013;381:752-62.

3 Choi J, Ahn A, Kim S, et al. Global prevalence of physical frailty by fried's criteria in community-dwelling elderly with national population-based surveys. J Am Med Dir Assoc 2015;16:548-50.

4 Cameron ID, Fairhall N, Langron C, et al. A multifactorial interdisciplinary intervention reduces frailty in older people: randomized trial. BMC Med 2013;11:1-10.

5 Reeves D, Pye S, Ashcroft DM, et al. The challenge of ageing populations and patient frailty: can primary care adapt? BMJ 2018;362:k3349.

6 Warmoth K, Lang IA, Phoenix C, et al. 'Thinking you're old and frail': a qualitative study of frailty in older adults. Ageing Soc 2016;36:1483-500.

7 Nicholson C, Gordon AL, Tinker A. Changing the way "we" view and talk about frailty.... Age Ageing 2017:46:349-51. ...

8 Nicholson C, Meyer J, Flatley M, et al. Living on the margin: understanding the experience of living and dying with frailty in old age. Soc Sci Med 2012;75:1426-32.

9 Nicholson C, Meyer J, Flatley M, et al. The experience of living at home with frailty in old age: a psychosocial qualitative study. Int $J$ Nurs Stud 2013;50:1172-9.

10 van Campen C. Frail older persons in the Netherlands. The Hague, Netherlands: The Netherlands Institute for Social Research, 2011. https://www.scp.nl/dsresource?objectid=baac1e00-8a53-4372-9df52b67e65a6991\&type =org

11 Shaw RL, Gwyther H, Holland C, et al. Understanding frailty: meanings and beliefs about screening and prevention across key stakeholder groups in Europe. Ageing Soc 2018;38:1223-52.

12 Schoenborn NL, Van Pilsum Rasmussen SE, Xue Q-L, et al. Older adults' perceptions and informational needs regarding frailty. BMC Geriatr 2018;18:1-7.

13 Archibald MM, Ambagtsheer R, Beilby J, et al. Perspectives of frailty and frailty screening: protocol for a collaborative knowledge translation approach and qualitative study of Stakeholder understandings and experiences. BMC Geriatr 2017;17:87.

14 Gilleard C, Higgs P. Aging without agency: theorizing the fourth age. Aging Ment Health 2010;14:121-8.

15 Grenier A. Transitions and the lifecourse: challenging the constructions of 'growing old'. London: Policy Press, 2012.
16 Skilbeck JK, Arthur A, Seymour J. Making sense of frailty: an ethnographic study of the experience of older people living with complex health problems. Int J Older People Nurs 2018;13:e12172-11.

17 Grenier A. Constructions of frailty in the English language, care practice and the lived experience. Ageing Soc 2007;27:425-45.

18 Hummert ML. Age stereotypes and aging. In: Schaie KW, Sherry LW, eds. Handbook of the psychology of aging. San Diego, California: Academic Press, 2011: 249-62.

19 Puts MTE, Shekary N, Widdershoven G, et al. The meaning of frailty according to Dutch older frail and non-frail persons. J Aging Stud 2009;23:258-66.

20 Grenier A. The distinction between being and feeling frail: exploring emotional experiences in health and social care. $J$ Soc Work Pract 2006;20:299-313.

21 Andrew MK, Fisk JD, Rockwood K. Psychological well-being in relation to frailty: a frailty identity crisis? Int Psychogeriatr 2012;24:1347-53.

22 Fillit H, Butler RN. The frailty identity crisis. J Am Geriatr Soc 2009;57:348-52.

23 Robertson DA, Kenny RA. Negative perceptions of aging modify the association between frailty and cognitive function in older adults. Pers Individ Dif 2016;100:120-5.

24 Warmoth K, Tarrant M, Abraham C, et al. Relationship between perceptions of ageing and frailty in English older adults. Psychol Health Med 2018;23:465-74.

25 Age UK and the British Geriatrics Society. Frailty: language and perceptions: a report prepared by BritainThinks on behalf of age UK and the British geriatrics Society. London: BritainThinks, 2015. http:// www.ageuk.org.uk/Documents/EN-GB/For-professionals/ Policy/ health-and-wellbeing/report_bgs_frailty_language_and_perceptions. pdf?dtrk=true

26 Archibald MM, Lawless MM, Ambagtsheer MM, et al. Consumer perspectives of frailty screening. In Submission.

27 Archibald MM, Lawless M, Gill TK, et al. Orthopaedic surgeons' perceptions of frailty and frailty screening. BMC Geriatr 2020;20:17.

28 C Ambagtsheer R, M Archibald M, Lawless M, et al. General practitioners' perceptions, attitudes and experiences of frailty and frailty screening. Aust J Gen Pract 2019;48:426-33.

29 Kitson A, Powell K, Hoon E, et al. Knowledge translation within a population health study: how do you do it? Implementation Science 2013:8.

30 Thorne S. Interpretive description. Walnut Creek, CA: Left Coast Press, 2008.

31 Thorne S, Kirkham SR, O'Flynn-Magee K. The analytic challenge in interpretive description. Int J Qual Methods 2004;3:1-11.

32 Morley JE, Vellas B, Abellan van Kan G, et al. Frailty consensus: a call to action. J Am Med Dir Assoc 2013;14:392-7.

33 Kaehr E, Visvanathan R, Malmstrom TK, et al. Frailty in nursing homes: the FRAIL-NH scale. J Am Med Dir Assoc 2015;16:87-9.

34 Braun V, Clarke V. Using thematic analysis in psychology. Qual Res Psychol 2006;3:77-101.

35 Fereday J, Muir-Cochrane E. Demonstrating rigor using thematic analysis: a hybrid approach of inductive and deductive coding and theme development. Int J Qual Methods 2006;5:80-92.

36 Taylor SE, Crocker J. Schematic bases of social information processing. In: Higgins ET, Bargh JA, eds. Social cognition. Hillsdale, NJ: Lawrence Erlbaum Associates, 1981: 89-134.

37 Weiss D, Lang FR. Thinking about my generation: adaptive effects of a dual age identity in later adulthood. Psychol Aging 2009;24:729-34.

38 Levy BR, Hausdorff JM, Hencke R, et al. Reducing cardiovascular stress with positive self-stereotypes of aging. J Gerontol B Psychol Sci Soc Sci 2000;55:P205-13.

39 Levy BR, Myers LM. Preventive health behaviors influenced by selfperceptions of aging. Prev Med 2004;39:625-9.

40 Levy BR, Slade MD, Kunkel SR, et al. Longevity increased by positive self-perceptions of aging. J Pers Soc Psychol 2002;83:261-70.

41 Lawless MT, Archibald MM, Ambagtsheer RC, et al. Factors influencing communication about frailty in primary care: a scoping review. Patient Educ Couns 2019. doi:10.1016/j.pec.2019.09.014. [Epub ahead of print: 16 Sep 2019].

42 Robertson DA, King-Kallimanis BL, Kenny RA. Negative perceptions of aging predict longitudinal decline in cognitive function. Psychol Aging 2016;31:71-81. 